\title{
Correction to "Menaquinone-4 Accelerates Calcification of Human Aortic Valve Interstitial Cells in High-Phosphate Medium through PXR"
}

\author{
In Figure 2A of the above article [Yang W, Yu Z, Chiyoya M, Liu X, Daitoku K, Motomura S, \\ Imaizumi T, Fukuda I, Furukawa K-I, Tsuji M, and Seya K (2020) J Pharmacol Exp Ther \\ 372(3): 277-284; DOI: https://doi.org/10.1124/jpet.119.263160], the authors discovered an \\ extra image was accidentally added to the top of panel A. The correct version of Figure 2 and \\ a corrected caption are provided below.
}

The HTML and PDF versions of the article have been corrected.

The authors apologize for any inconvenience caused by this error.

A

C
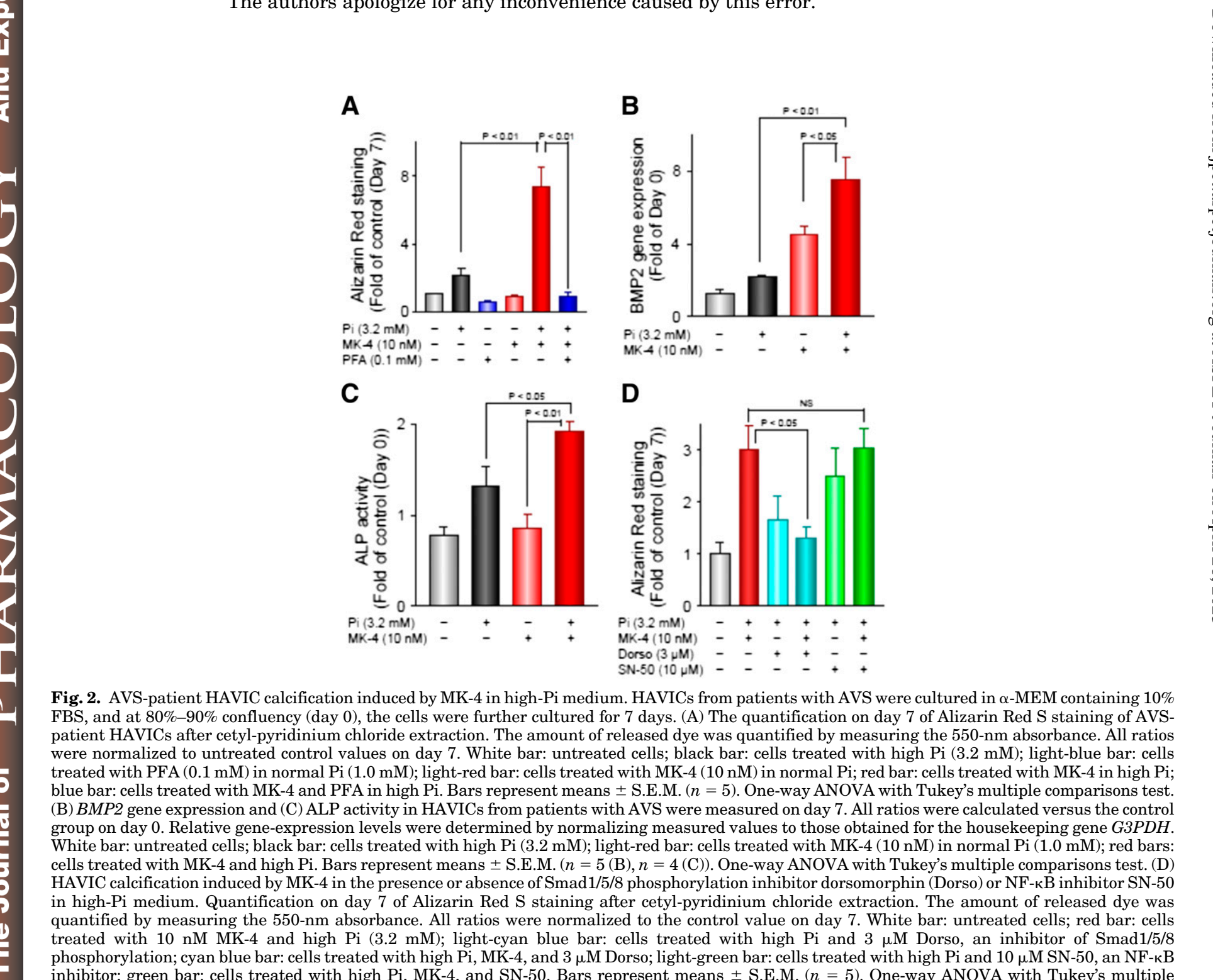

B

D
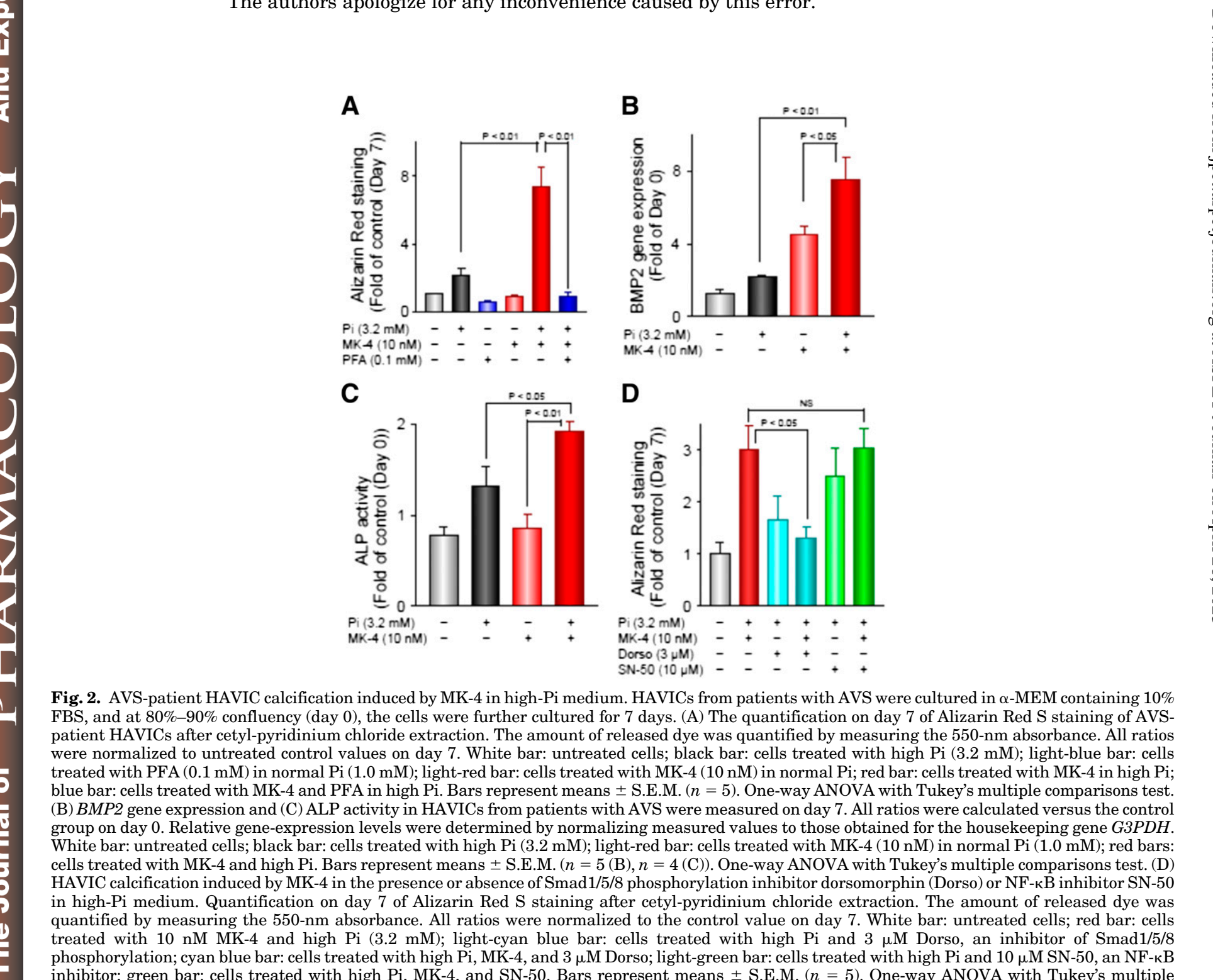

Fig. 2. AVS-patient HAVIC calcification induced by MK-4 in high-Pi medium. HAVICs from patients with AVS were cultured in $\alpha$-MEM containing $10 \%$ FBS, and at 80\%-90\% confluency (day 0), the cells were further cultured for 7 days. (A) The quantification on day 7 of Alizarin Red S staining of AVSpatient HAVICs after cetyl-pyridinium chloride extraction. The amount of released dye was quantified by measuring the 550 -nm absorbance. All ratios were normalized to untreated control values on day 7. White bar: untreated cells; black bar: cells treated with high Pi (3.2 mM); light-blue bar: cells treated with PFA $(0.1 \mathrm{mM})$ in normal Pi $(1.0 \mathrm{mM})$; light-red bar: cells treated with MK-4 $(10 \mathrm{nM})$ in normal Pi; red bar: cells treated with MK-4 in high Pi; (B) (B) BMP2 gene expression and (C) ALP activity in HAVICs from patients with AVS were measured on day 7. All ratios were calculated versus the control group on day 0 . Relative gene-expression levels were determined by normalizing measured values to those obtained for the housekeeping gene G3PDH. White bar: untreated cells; black bar: cells treated with high Pi (3.2 mM); light-red bar: cells treated with MK-4 (10 nM) in normal Pi (1.0 mM); red bars: cells treated with MK-4 and high Pi. Bars represent means \pm S.E.M. $(n=5(\mathrm{~B}), n=4$ (C)). One-way ANOVA with Tukey's multiple comparisons test. (D)

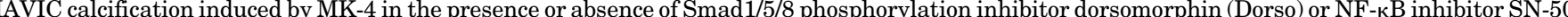
$\mathrm{n}$ high-Pi medium. Quantification on day 7 of Alizarin Red S staining after cetyl-pyridinium chloride extraction. The amount of released dye was in 550 -nm absorbance. All ratios were normalized to the control value on day 7 . White bar: untreated cells; red bar: cells phosphorylation; cyan blue bar: cells treated with high Pi, MK-4, and $3 \mu \mathrm{M}$ Dorso; light-green bar: cells treated with high Pi and 10 $\mu$ M SN-50, an NF-кB inhibitor; green bar: cells treated with high Pi, MK-4, and SN-50. Bars represent means \pm S.E.M. $(n=5)$. One-way ANOVA with Tukey's multiple comparisons test. 